CARPATHIAN J. MATH.

Volume 37 (2021), No. 3,

Pages $529-540$

\title{
Approximate optimality and approximate duality in nonsmooth composite vector optimization
}

\author{
THANATCHAPORN SIRICHUNWIJIT ${ }^{1}$, RABIAN WANGKEEREE ${ }^{1,2}$ and NitHIRAT SiSARAT ${ }^{1}$
}

\begin{abstract}
This paper concentrates on studying a nonsmooth composite vector optimization problem (P for brevity). We employ a fuzzy necessary condition for approximate (weakly) efficient solutions of a nonconvex and nonsmooth cone constrained vector optimization problem established in [Choung, T. D. Approximate solutions in nonsmooth and nonconvex cone constrained vector optimization Ann. Oper. Res. (2020), https://doi.org/10.1007/s10479-020-03740-3.] and the a chain rule for generalized differentiation to provide a necessary condition which exhibited in a Fritz-John type for approximate (weakly) efficient solutions of (P). Sufficient optimality conditions for approximate (weakly) efficient solutions to $(\mathrm{P})$ are also provided by means of proposing the use of (strictly) approximately generalized convex composite vector functions with respect to a cone. Moreover, an approximate dual vector problem to $(\mathrm{P})$ is given and strong and converse duality assertions for approximate (weakly) efficient solutions are proved.
\end{abstract}

\section{INTRODUCTION}

Due to the increased complexity of the optimization problems that have many questions of theoretical and computational interest, the study of problems that encompass as special instances of the already remedied ones are of great concern. In this paper, we lay out a unified framework for examining optimization problems by viewing a composite vector optimization problem of the form:

$$
\min _{K}\{(f \circ F)(x) \mid x \in \Omega,(g \circ G)(x) \in-S\},
$$

where $F: X \rightarrow W, G: X \rightarrow V, f: W \rightarrow Y$ and $g: V \rightarrow Z$ are vector functions between finite-dimensional spaces, $K \subset Y$ is a pointed (i.e., $K \cap(-K)=\{0\}$ ) closed convex cone, $S \subset Z$ is a nonempty closed convex cone and $\Omega \subset X$ is a nonempty closed set. Hereafter, we always assume that the topological interior of $K$ is nonempty (i.e., int $K \neq \emptyset$ ) and $F, G, f, g$ are locally Lipschitz at the corresponding points under consideration. The modeling of problems as $(\mathrm{P})$ covers broad classes of various optimization problems such as conic vector optimization problems, (standard) multiobjective/vector optimization problems, multiobjective approximation problems [8, 23, 42], and many optimization problems manufactured in practical fields like engineering or economics and finance, Over the last couple of decades, issues related to optimality conditions and duality for (weakly) efficient solutions of the model problem $(\mathrm{P})$ have been extensively investigated in the literature; see $[1,2,7,8,23,31,32,36]$ and other references therein. For other results concerning on optimality conditions and duality in both smooth/nonsmooth multiobjective/vector optimization problems involving convex/generalized convex functions, we refer the readers to $[3,5,6,24,25,38,41]$ and other references therein.

Received: 12.10.2020. In revised form: 25.06.2021. Accepted: 02.07.2021

2010 Mathematics Subject Classification. 49K99, 90C46, 90C29, 65K10 .

Key words and phrases. approximate composite vector optimization, optimality conditions, duality, generalized convexity, Limiting/Mordukhovich subdifferential.

Corresponding author: Rabian Wangkeeree; rabianw@nu.ac.th 
As known to all, from the computational point of view, approximate solutions in optimization problems occur naturally by way of stopping numerical procedures after a finite number of steps. Moreover, in general, optimization problems do not necessarily have the exact solutions whereas approximate ones exist under very mild hypotheses. Therefore, it is significant to study approximate solutions instead to optimization problems from both points of view, and so, many authors have turned their more and more attention to this topic; see [10,12, 15, 16, 17, 18, 19, 20, 21, 26, 27, 28, 40] and the references therein. The interested reader is referred to $[9,13,14]$ for more information on optimality conditions and duality for approximate (weakly) efficient solutions in connection with the multiobjective/vector optimization. With this aim in view, in this paper, we continue a broad framework for examining approximate (weakly) efficient solutions for the problem $(\mathrm{P})$ which, to our knowledge, have not been investigated yet. It should be noted here that the most notion of approximate solutions for optimization problems involving nonconvex functions is that of approximate-quasi solutions which can be regarded as a local concepts of approximate solutions in view of Ekeland's variational principle [11]. This notion has also been extended to vector optimization problems, see e.g., [26, 28]. In what follows, let us now recall the concept of (weak) $e$-quasi efficient solutions for the problem (P).

Definition 1.1. Let $e \in K$ and $\bar{x} \in C:=\{x \in \Omega \mid(g \circ G)(x) \in-S\}$.

(i) One say that $\bar{x}$ is a weak $e$-quasi efficient solution of problem $(\mathrm{P})$, denoted by $\bar{x} \in e-\mathcal{S}^{w}(\mathrm{P})$, whenever

$$
\forall x \in C,(f \circ F)(x)-(f \circ F)(\bar{x})+\|x-\bar{x}\| e \notin-\operatorname{int} K .
$$

(ii) One say that $\bar{x}$ is an $e$-quasi efficient solution of problem $(\mathrm{P})$, denoted by $\bar{x} \in$ $e-\mathcal{S}(\mathrm{P})$, whenever

$$
\forall x \in C,(f \circ F)(x)-(f \circ F)(\bar{x})+\|x-\bar{x}\| e \notin-K \backslash\{0\} .
$$

Note also that in case of $e:=0$, the above-defined (weak) $e$-quasi efficient solution collapses to (weak) Pareto solution defined as in [22, 29, 39].

The aim of this paper is to study a class of approximate (weakly) efficient solutions, i.e., (weak) $e$-quasi efficient solutions to a nonsmooth composite vector optimization problem (P). We apply a fuzzy necessary condition [9, Theorem 3.2] for approximate (weakly) efficient solutions of a nonconvex and nonsmooth cone constrained vector optimization problem, which was recently established based on the approximate extremal principle and the a chain rule for generalized differentiation $[34,35]$ to achieve a necessary condition which exhibited in a Fritz-John type for approximate (weakly) efficient solutions of $(\mathrm{P})$. This formulation is expressed in terms of the limiting/Mordukhovich subdifferential. Sufficient optimality conditions for approximate (weakly) efficient solutions to $(\mathrm{P})$ are also provided by means of proposing the use of (strictly) approximately generalized convex composite vector functions with respect to a cone. According to approximate optimality conditions, we state an approximate dual vector problem to $(\mathrm{P})$ and explore strong and converse duality assertions for approximate (weakly) efficient solutions.

The rest of paper is organized as follows. The next section presents some notations and preliminaries. Sect. 3 is devoted to establishing necessary and sufficient optimality conditions for a approximate (weakly) efficient solutions of a nonsmooth composite vector optimization problem (P). Finally, Sect. 4 explores duality relations between approximate (weak) efficient solutions of the problem (P) and its dual one in the sense of Mond and Weir. 


\section{PRELIMINARIES}

In this section, we recall some notations, basic definitions, and preliminary results which will be utilized throughout the paper. Now, all spaces are assumed to be finitedimensional equipped with norms $\|\cdot\|$. A closed unit ball in $X$ is denoted by $B_{X}$. The topological closure and the topological interior of a set $\Omega \subset X$ are denoted by $\mathrm{cl} \Omega$ and int $\Omega$, respectively. As usual, the dual cone of $\Omega \subset X$ is the set $\Omega^{+}:=\left\{x^{*} \in X \mid\left\langle x^{*}, x\right\rangle \geq\right.$ $0, \forall x \in \Omega\}$. Also, for $n \in \mathbb{N}:=\{1,2, \ldots\}$, we denote by $\mathbb{R}_{+}^{n}$ the nonnegative orthant of $\mathbb{R}^{n}$. For a product space $X \times Y$, its norm is defined by $\|(x, y)\|=\|x\|+\|y\|$ for $x \in X$ and $y \in Y$.

Given a set-valued mapping (or multifunctions) $H: X \rightrightarrows X$, with values $H(x) \subset X$ in the collection of all the subsets of $X$, we denote by

$$
\limsup _{x \rightarrow \bar{x}} H(x):=\left\{x^{*} \in X \mid \exists\left\{x_{n}\right\} \rightarrow x_{0}, x_{n}^{*} \rightarrow x^{*} \text { with } x_{n}^{*} \in H\left(x_{n}\right) \text { for all } n \in \mathbb{N}\right\} .
$$

the sequential Painlevé-Kuratowski upper/outer limit of $H$ as $x \rightarrow \bar{x}$.

Let $\Omega \subset X$ be closed around $x \in \Omega$, i.e., there is a neighborhood $U$ of $\bar{x}$ such that $\Omega \cap \mathrm{cl} U$ is closed. The Fréchet normal cone to $\Omega$ at $x \in \Omega$ is defined by

$$
\widehat{N}(\bar{x} ; \Omega):=\left\{\begin{array}{l|l}
x^{*} \in X \mid \limsup _{x \stackrel{\Omega}{\longrightarrow} \bar{x}} \frac{\left\langle x^{*}, x-\bar{x}\right\rangle}{\|x-\bar{x}\|} \leq 0
\end{array}\right\},
$$

where $x \stackrel{\Omega}{\rightarrow} \bar{x}$ stands for $x \rightarrow \bar{x}$ with $x \in \Omega$. If $x \notin \Omega$, we stipulate that $\widehat{N}(x ; \Omega):=\emptyset$.

The Mordukhovich/limiting normal cone $N(\bar{x} ; \Omega)$ to $\Omega$ at $\bar{x} \in \Omega$ is obtained from Fréchet normal cones by taking the sequential Painlevé-Kuratowski upper limits as:

$$
N(\bar{x} ; \Omega):=\limsup _{x \stackrel{\Omega}{\rightarrow} \bar{x}} \widehat{N}(x ; \Omega) .
$$

If $x \notin \Omega$, we put $N(x ; \Omega):=\emptyset$.

For an extended real-valued function $\varphi: X \rightarrow \overline{\mathbb{R}}:=\mathbb{R} \cup\{+\infty\}$, the effective domain and the epigraph are respectively defined by

$$
\operatorname{dom} \varphi:=\{x \in X \mid \varphi(x)<+\infty\} \text { and epi } \varphi:=\{(x, \mu) \in X \times \mathbb{R} \mid \mu \geq \varphi(x)\} .
$$

The Mordukhovich/limiting subdifferential and the Fréchet subdifferential of $\varphi$ at $\bar{x} \in \operatorname{dom} \varphi$ are defined, respectively, by

$$
\partial \varphi(\bar{x}):=\left\{x^{*} \in X \mid\left(x^{*},-1\right) \in N((\bar{x}, \varphi(\bar{x})) ; \text { epi } \varphi)\right\},
$$

and

$$
\widehat{\partial} \varphi(\bar{x}):=\left\{x^{*} \in X \mid\left(x^{*},-1\right) \in \widehat{N}((\bar{x}, \varphi(\bar{x})) ; \text { epi } \varphi)\right\} .
$$

If $\bar{x} \notin \operatorname{dom} \varphi$, then one puts $\partial \varphi(\bar{x}):=\widehat{\partial} \varphi(\bar{x}):=\emptyset$. It is worth noting [34,35] that if $\varphi$ is a convex function, the above-defined subdifferential coincides with the subdifferential in the sense of convex analysis [37].

For any vector function $f: X \rightarrow Y$ we can associate $f$ with a scalarization function with respect to some $y^{*} \in Y$ defined by

$$
\left\langle y^{*}, f\right\rangle(x):=\left\langle y^{*}, f(x)\right\rangle, x \in X .
$$

We close the section by the following results that are needed for our study.

Lemma 2.1. Let $y^{*} \in \mathbb{R}^{n}$, and let $f: X \rightarrow \mathbb{R}^{n}$ be Lipschitz continuous around $\bar{x} \in X$. We have

(i) [34, Proposition 3.5] $x^{*} \in \widehat{\partial}\left\langle y^{*}, f\right\rangle(\bar{x}) \Leftrightarrow\left(x^{*},-y^{*}\right) \in \widehat{N}((\bar{x}, f(\bar{x})) ; g p h f)$.

(ii) [34, Theorem 1.90] $x^{*} \in \partial\left\langle y^{*}, f\right\rangle(\bar{x}) x \Leftrightarrow\left(x^{*},-y^{*}\right) \in N((\bar{x}, f(\bar{x})) ; g p h f)$. 
Lemma 2.2. [34, Corollary 3.43] Let $f: X \rightarrow Y$ be locally Lipschitz at $\bar{x} \in X$, and let $\varphi: Y \rightarrow \mathbb{R}$ be locally Lipschiz around $f(\bar{x})$. Then one has

$$
\partial(\varphi \circ f)(\bar{x}) \subset \bigcup_{y^{*} \in \partial \varphi(f(\bar{x}))} \partial\left\langle y^{*}, f\right\rangle(\bar{x}) .
$$

\section{APPROXIMATE OPTIMALITY CONDITIONS IN COMPOSITE VECTOR OPTIMIZATION}

The aim of this section is to devote to studying necessary and sufficient optimality conditions for (weak) $e$-quasi efficient solutions of problem (P). The forthcoming theorem provides a Fritz-John type necessary optimality condition, expressed in terms of the limiting subdifferential, for (weak) $e$-quasi efficient solutions of problem (P); the proof is motivated by [8, Theorem 3.1] and [9, Theorem 3.2]. To this aim, we need a fuzzy necessary optimality condition for (weak) e-quasi efficient solutions in conic vector optimization problems as follows.

Lemma 3.3. For the problem $(\mathrm{P})$ with $X=W=V$ and $F$ and $G$ are identical maps, let $\bar{x} \in$ $e-\mathcal{S}^{w}(\mathrm{P})$. Then, for a given $k \in \mathbb{N}$, one can find $x^{1 k} \in B_{X}\left(\bar{x}, \frac{1}{k}\right), x^{2 k} \in B_{X}\left(\bar{x}, \frac{1}{k}\right), x^{3 k} \in$ $\Omega \cap B_{X}\left(\bar{x}, \frac{1}{k}\right), y_{k}^{*} \in K^{+}$and $z_{k}^{*} \in S^{+}$such that $\left\|y_{k}^{*}\right\|=1$ and

$$
0 \in \widehat{\partial}\left\langle y_{k}^{*}, f\right\rangle\left(x^{1 k}\right)+\widehat{\partial}\left\langle z_{k}^{*}, g\right\rangle\left(x^{2 k}\right)+\widehat{N}\left(x^{3 k} ; \Omega\right)+\left(\left\langle y_{k}^{*}, e\right\rangle+\frac{1}{k}\right) B_{X},\left|\left\langle z_{k}^{*}, g\left(x^{2 k}\right)\right\rangle\right| \leq \frac{1}{k} .
$$

Theorem 3.1. Let $\bar{x} \in e-\mathcal{S}^{w}(\mathrm{P})$. Then, there exist $y^{*} \in K^{+}$and $z^{*} \in S^{+}$with $\left\|y^{*}\right\|+\left\|z^{*}\right\|=1$, such that

$$
\left\{\begin{array}{l}
0 \in \bigcup_{w^{*} \in \partial\left\langle y^{*}, f\right\rangle(F(\bar{x}))} \partial\left\langle w^{*}, F\right\rangle(\bar{x})+\bigcup_{v^{*} \in \partial\left\langle z^{*}, g\right\rangle(G(\bar{x}))} \partial\left\langle v^{*}, G\right\rangle(\bar{x})+\left\langle y^{*}, e\right\rangle B_{X}+N(\bar{x} ; \Omega), \\
\left\langle z^{*}, g(G(\bar{x}))=0 .\right.
\end{array}\right.
$$

Proof. We begin by putting $\tilde{f}=f \circ F$ and $\tilde{g}=g \circ G$. On account of $\bar{x} \in e-\mathcal{S}^{w}(\mathrm{P})$, we invoke Lemma 3.3 to assert that for each $k \in \mathbb{N}$ there exist $x^{1 k} \in B_{X}\left(\bar{x}, \frac{1}{k}\right), x^{2 k} \in B_{X}\left(\bar{x}, \frac{1}{k}\right)$, $x^{3 k} \in \Omega \cap B_{X}\left(\bar{x}, \frac{1}{k}\right), y_{k}^{*} \in K^{+}$with $\left\|y_{k}^{*}\right\|=1$ and $z_{k}^{*} \in S^{+}$such that

$$
0 \in \widehat{\partial}\left\langle y_{k}^{*}, \tilde{f}\right\rangle\left(x^{1 k}\right)+\widehat{\partial}\left\langle z_{k}^{*}, \tilde{g}\right\rangle\left(x^{2 k}\right)+\widehat{N}\left(x^{3 k} ; \Omega\right)+\left(\left\langle y_{k}^{*}, e\right\rangle+\frac{1}{k}\right) B_{X},\left|\left\langle z_{k}^{*}, \tilde{g}\left(x^{2 k}\right)\right\rangle\right| \leq \frac{1}{k} .
$$

Consequently, we find sequences $\left\{x^{1 k}\right\} \subset X,\left\{x^{2 k}\right\} \subset X,\left\{x^{3 k}\right\} \subset X,\left\{x_{1 k}^{*}\right\} \subset X,\left\{x_{2 k}^{*}\right\} \subset$ $X,\left\{x_{3 k}^{*}\right\} \subset X,\left\{y_{k}^{*}\right\} \subset K^{+}$with $\left\|y_{k}^{*}\right\|=1$ and $\left\{z_{k}^{*}\right\} \subset S^{+}$such that $x_{1 k}^{*} \in \widehat{\partial}\left\langle y_{k}^{*}, f \circ F\right\rangle\left(x^{1 k}\right)$, $x_{2 k}^{*} \in \widehat{\partial}\left\langle z_{k}^{*}, g \circ G\right\rangle\left(x^{2 k}\right), x_{3 k}^{*} \in \widehat{N}\left(x^{3 k} ; \Omega\right)$,

$$
0 \in x_{1 k}^{*}+x_{2 k}^{*}+x_{3 k}^{*}+\left(\left\langle y_{k}^{*}, e\right\rangle+\frac{1}{k}\right) B_{X},
$$

and

$$
x^{1 k} \rightarrow \bar{x}, x^{2 k} \rightarrow \bar{x}, x^{3 k} \rightarrow \bar{x},\left\langle z_{k}^{*}, g \circ G\right\rangle\left(x^{2 k}\right) \rightarrow 0 \text { as } k \rightarrow \infty .
$$

Let us note by passing to a subsequence if necessary that $y_{k}^{*} \rightarrow \tilde{y}^{*} \in K^{+}$as $k \rightarrow \infty$, where $\left\|\tilde{y}^{*}\right\|=1$. By our assumptions, we suppose that $f \circ F$ is locally Lipschitz at $\bar{x}$ with a modulus $l_{1}>0$. It then follows from $x_{1 k}^{*} \in \widehat{\partial}\left\langle y_{k}^{*}, f \circ F\right\rangle\left(x^{1 k}\right)$ together with [34, Proposition 1.85] that $\left\|x_{1 k}^{*}\right\| \leq l_{1}\left\|y_{k}^{*}\right\|=l_{1}$ for all $k \in \mathbb{N}$. Hence, as $X$ is a finite-dimensional space, we may assume by taking a subsequence if necessary that $x_{1 k}^{*} \rightarrow x_{1}^{*} \in X$ as $k \rightarrow \infty$. Similarly, let $l_{2}>0$ be a Lipschitz constant of $g \circ G$ around $\bar{x}$, and so,

$$
\left\|x_{2 k}^{*}\right\| \leq l_{2}\left\|z_{k}^{*}\right\|, \forall k \in \mathbb{N} .
$$

Let us now consider two the following cases: 
(C1): Assume that $\left\{z_{k}^{*}\right\}$ is unbounded. There is no loss of generality in assuming that $\left\|z^{*}\right\| \rightarrow \infty$ and $\frac{z_{k}^{*}}{\left\|z_{k}^{*}\right\|} \rightarrow z^{*} \in S^{+}$with $\left\|z^{*}\right\|=1$ as $k \rightarrow \infty$. It stems from (3.5) we have $\left\langle\frac{z_{k}^{*}}{\left\|z_{k}^{*}\right\|},(g \circ G)\left(x^{2 k}\right)\right\rangle \rightarrow 0$ as $k \rightarrow \infty$, which in turn gives us the equality $\left\langle z^{*}, g(G(\bar{x}))\right\rangle=0$. In addition, by (3.6), we may assume that $\frac{x_{2 k}^{*}}{\left\|z_{k}^{*}\right\|} \rightarrow x_{2}^{*} \in X$ as $k \rightarrow \infty$. Then, in view of (3.4), there exist $b_{k} \in B_{X}, k \in \mathbb{N}$ such that

$$
-\frac{x_{1 k}^{*}}{\left\|z_{k}^{*}\right\|}-\frac{x_{2 k}^{*}}{\left\|z_{k}^{*}\right\|}-\frac{\left(\left\langle y_{k}^{*}, e\right\rangle+\frac{1}{k}\right) b_{k}}{\left\|z_{k}^{*}\right\|}=\frac{x_{3 k}^{*}}{\left\|z_{k}^{*}\right\|} \in \widehat{N}\left(x^{3 k} ; \Omega\right), k \in \mathbb{N} .
$$

Letting $k \rightarrow \infty$ in (3.7) and noticing (2.1), we get

$$
-x_{2}^{*} \in N(\bar{x} ; \Omega) \text {. }
$$

According to Lemma 2.1(i), for each $k \in \mathbb{N}$, the inclusion $x_{2 k}^{*} \in \widehat{\partial}\left\langle z_{k}^{*}, g \circ G\right\rangle\left(x^{2 k}\right)$ amounts to $\left(x_{2 k}^{*},-z_{k}^{*}\right) \in \widehat{N}\left(\left(x^{2 k},(g \circ G)\left(x^{2 k}\right)\right)\right.$; gph $\left.(g \circ G)\right)$, and consequently, $\left(\frac{x_{2 k}^{*}}{\left\|z_{k}^{*}\right\|}, \frac{-z_{k}^{*}}{\left\|z_{k}^{*}\right\|}\right) \in \widehat{N}\left(\left(x^{2 k},(g \circ\right.\right.$ $\left.G)\left(x^{2 k}\right)\right)$; gph $\left.(g \circ G)\right)$. Passing to the limit as $k \rightarrow \infty$ in the preceding inclusion and taking (2.1) into account, we obtain that $\left(x_{2}^{*},-z^{*}\right) \in N((\bar{x},(g \circ G)(\bar{x}))$; gph $(g \circ G))$, which, by virtue of 2.1(ii), is equivalent to

$$
x_{2}^{*} \in \partial\left\langle z^{*}, g \circ G\right\rangle(\bar{x}) .
$$

Combining (3.8) along with (3.9) and by taking $y^{*}:=0 \in K^{+}$, we arrive at

$$
0 \in \partial\left\langle y^{*}, f \circ F\right\rangle(\bar{x})+\partial\left\langle z^{*}, g \circ G\right\rangle(\bar{x})+\left\langle y^{*}, e\right\rangle B_{X}+N(\bar{x} ; \Omega) .
$$

(C2): If $\left\{z_{k}^{*}\right\}$ is bounded, then we may assume by taking a subsequence if necessary that $z_{k}^{*} \rightarrow \tilde{z}^{*} \in S^{+}$as $k \rightarrow \infty$. Due to (3.6), we also have that $\left\{x_{2 k}^{*}\right\}$ is bounded and so, we may assume without loss of generality that $x_{2 k}^{*} \rightarrow x_{2}^{*} \in X$ as $k \rightarrow \infty$. As above, we get from the inclusion $x_{1 k}^{*} \in \widehat{\partial}\left\langle y_{k}^{*}, f \circ F\right\rangle\left(x^{1 k}\right)$ that $\left(x_{1 k}^{*},-y_{k}^{*}\right) \in \widehat{N}\left(\left(x^{1 k},(f \circ F)\left(x^{1 k}\right)\right)\right.$; gph $\left.(f \circ F)\right)$ for all $k \in \mathbb{N}$. Therefore,

$$
\left(\frac{x_{1 k}^{*}}{\left\|y_{k}^{*}\right\|+\left\|z_{k}^{*}\right\|}, \frac{-y_{k}^{*}}{\left\|y_{k}^{*}\right\|+\left\|z_{k}^{*}\right\|}\right) \in \widehat{N}\left(\left(x^{1 k},(f \circ F)\left(x^{1 k}\right)\right) ; \operatorname{gph}(f \circ F)\right) \text {. }
$$

Passing (3.11) to the limit as $k \rightarrow \infty$ and noticing (2.1), we obtain that

$$
\left(\frac{x_{1}^{*}}{\left\|\tilde{y}^{*}\right\|+\left\|\tilde{z}^{*}\right\|}, \frac{-\tilde{y}^{*}}{\left\|\tilde{y}^{*}\right\|+\left\|\tilde{z}^{*}\right\|}\right) \in N((\bar{x},(f \circ F)(\bar{x})) ; \operatorname{gph}(f \circ F)),
$$

which amounts to

$$
\frac{x_{1}^{*}}{\left\|\tilde{y}^{*}\right\|+\left\|\tilde{z}^{*}\right\|} \in \partial\left\langle y^{*}, f \circ F\right\rangle(\bar{x})
$$

where $y^{*}:=\frac{\tilde{y}^{*}}{\left\|\tilde{y}^{*}\right\|+\left\|\tilde{z}^{*}\right\|}$. Similarly, we obtain that $\left\langle z^{*}, g \circ G(\bar{x})\right\rangle=0$ and

$$
\frac{x_{2}^{*}}{\left\|\tilde{y}^{*}\right\|+\left\|\tilde{z}^{*}\right\|} \in \partial\left\langle z^{*}, g \circ G\right\rangle(\bar{x})
$$

where $z^{*}:=\frac{\tilde{z}^{*}}{\left\|\tilde{y}^{*}\right\|+\left\|\tilde{z}^{*}\right\|}$. On the one hand, (3.4) yields that there exist $b_{k} \in B_{X}, k \in \mathbb{N}$ such that

$$
-\frac{x_{1 k}^{*}}{\left\|y_{k}^{*}\right\|+\left\|z_{k}^{*}\right\|}-\frac{x_{2 k}^{*}}{\left\|y_{k}^{*}\right\|+\left\|z_{k}^{*}\right\|}-\frac{\left(\left\langle y_{k}^{*}, e\right\rangle+\frac{1}{k}\right) b_{k}}{\left\|y_{k}^{*}\right\|+\left\|z_{k}^{*}\right\|}=\frac{x_{3 k}^{*}}{\left\|y_{k}^{*}\right\|+\left\|z_{k}^{*}\right\|} \in \widehat{N}\left(x^{3 k} ; \Omega\right), k \in \mathbb{N} .
$$

Also, we may assume by passing to subsequences if necessary that $b_{k} \rightarrow b \in B_{X}$ as $k \rightarrow \infty$. Now, letting $k \rightarrow \infty$ in (3.14) and noticing (2.1), we get the relation

$$
-\frac{x_{1}^{*}}{\left\|\tilde{y}^{*}\right\|+\left\|\tilde{z}^{*}\right\|}-\frac{x_{2}^{*}}{\left\|\tilde{y}^{*}\right\|+\left\|\tilde{z}^{*}\right\|}-\frac{\left\langle\tilde{y}^{*}, e\right\rangle b}{\left\|\tilde{y}^{*}\right\|+\left\|\tilde{z}^{*}\right\|} \in N(\bar{x} ; \Omega) .
$$


Combining this with (3.12) and (3.13), (3.10) also holds.

Now, let us note that $F$ and $G$ are locally Lipschitz at $\bar{x}$, by our assumptions, and $\left\langle y^{*}, f\right\rangle$ and $\left\langle z^{*}, g\right\rangle$ are locally Lipschitz at $F(\bar{x})$ and $G(\bar{x})$, respectively. Invoking (3.10) together with the chain rule (2.2) with $\varphi:=\left\langle y^{*}, f\right\rangle$ and $\psi:=\left\langle z^{*}, g\right\rangle$ proves that (3.3) is valid, and the proof is complete.

Let us now illustrate the usefulness of Theorem 3.1 for verifying necessary conditions for (weak) $e$-quasi efficient solutions of a substantial vector optimization problem via the following example, which is motivated by [9, Example 3.5].

Example 3.1. Consider the problem (P) with $\Omega:=\mathbb{R}, X:=\mathbb{R}, K:=\mathbb{R}_{+}^{2} \subset Y:=\mathbb{R}^{2}, S:=$ $\left\{\left(y_{1}, y_{2}\right) \in \mathbb{R}^{2}\left|y_{1} \geq\right| y_{2} \mid\right\} \subset Z:=\mathbb{R}^{2}, F(x):=\left(x^{2}, x+1\right), x \in \mathbb{R}, f(w):=\left(-\frac{1}{2} w_{1}+1,\left|w_{2}\right|\right)$, $w:=\left(w_{1}, w_{2}\right) \in \mathbb{R}^{2}, G(x):=x+1, x \in \mathbb{R}$, and $g(v):=\left(\frac{1}{2} v^{2}-v, v\right), v \in \mathbb{R}$. Let us notice that $C=\left\{v \in \mathbb{R}\left|\frac{1}{2}-\frac{1}{2} v^{2} \geq\right| v+1 \mid\right\}$, and let us select $\bar{x}:=-1 \in C$ and consider $e:=\left(e_{1}, e_{2}\right) \in K$. We can verify by definition that $\bar{x} \in e-S^{w}(P)$. By Taking $y^{*}:=$ $\left(\frac{1}{\sqrt{2}}, \frac{1}{\sqrt{2}}\right)$ and $z^{*}:=(0,0)$. Then, $y^{*} \in K^{+}, z^{*} \in S^{+}$satisfying $\left\|y^{*}\right\|+\left\|z^{*}\right\|=1$ and that $\partial\left\langle y^{*}, f\right\rangle(F(\bar{x}))=\left\{\left(-\frac{1}{2 \sqrt{2}}, \frac{1}{\sqrt{2}} x^{*}\right): x^{*} \in B_{X}\right\}, \partial\left\langle z^{*}, g\right\rangle(G(\bar{x}))=\{0\}$, $\bigcup_{w^{*} \in \partial\left\langle y^{*}, f\right\rangle(F(\bar{x}))} \partial\left\langle w^{*}, F\right\rangle(\bar{x})=\left\{\frac{1}{\sqrt{2}}\right\}+\frac{1}{\sqrt{2}} B_{X}, \bigcup_{v^{*} \in \partial\left\langle z^{*}, g\right\rangle(G(\bar{x}))} \partial\left\langle v^{*}, G\right\rangle(\bar{x})=\{0\}$ and $N(\bar{x} ; \Omega)=\{0\}$. It can be verified that the Fritz-John necessary condition (3.1) in Theorem 3.1 holds.

The following corollary provides a Fritz-John necessary condition for weak Pareto efficiencies of problem (P). This result develops [8, Theorem 3.2 ] and [9, Theorem 3.4] by letting $\Omega:=X$.

Corollary 3.1. Let $\bar{x} \in X$ be a weak Pareto solution of problem (P). Then, there exist $y^{*} \in K^{+}$ and $z^{*} \in S^{+}$with $\left\|y^{*}\right\|+\left\|z^{*}\right\|=1$, such that

$$
\left\{\begin{array}{l}
0 \in \bigcup_{w^{*} \in \partial\left\langle y^{*}, f\right\rangle(F(\bar{x}))} \partial\left\langle w^{*}, F\right\rangle(\bar{x})+\bigcup_{v^{*} \in \partial\left\langle z^{*}, g\right\rangle(G(\bar{x}))} \partial\left\langle v^{*}, G\right\rangle(\bar{x})+N(\bar{x} ; \Omega), \\
\left\langle z^{*}, g(G(\bar{x}))\right\rangle=0 .
\end{array}\right.
$$

Proof. Invoking Theorem 3.1 with $e:=0$, we obtain the desired result.

Remark 3.1. Corollary 3.1 reduces to [8, Corollary 3.5]. More exactly, the Clarke subdifferential of $f_{k}, k \in K$, and $g_{i}, i \in I$, at the considered point in framework of [30, Theorem 3.1].

Before we discuss the sufficient conditions for (weak) $e$-quasi efficient solutions of problem $(\mathrm{P})$, let us first define an approximate KKT condition for this problem.

Definition 3.2. Let $e \in K$ and let $\bar{x} \in C:=\{x \in \Omega \mid(g \circ G)(x) \in-S\}$. One says that $\bar{x}$ is said to satisfy the e-approximate KKT condition of problem (P) if (3.3) holds with $y^{*} \neq 0$.

Remark 3.2. In view of Theorem 3.1, observe that a weak e-quasi efficient solution $\bar{x}$ satisfies the above-defined approximate KKT condition solution under the fulfillment of the following constraint qualification: there does not exist $z^{*} \in S^{+}$with $\left\|z^{*}\right\|=1$ and $\left\langle z^{*}, g(G(\bar{x}))\right\rangle=0$, such that

$$
0 \in \bigcup_{v^{*} \in \partial\left\langle z^{*}, g\right\rangle(G(\bar{x}))} \partial\left\langle v^{*}, G\right\rangle(\bar{x})+N(\bar{x} ; \Omega) .
$$

It is noteworthy, however, that a feasible point at which the $e$-approximate KKT condition holds needs not be a (weak) e-quasi efficient solution in general; see e.g., [4, Example 3.14] in the case of $K:=\mathbb{R}_{+}^{3}, \Omega:=\mathbb{R}$, and $F$ and $G$ are identical maps. This fact leads us to employ the following notions of (strictly) approximately generalized convexity (with respect to a cone) for composite vector functions $F$ and $G$. 


\section{Definition 3.3.}

(i) We say that $(f \circ F, g \circ G)$ is $(K \times S)$-approximately generalized convex on $\Omega$ at $\bar{x} \in \Omega$ if for any $x \in \Omega, y^{*} \in K^{+}, z^{*} \in S^{+},\left\|y^{*}\right\|+\left\|z^{*}\right\|=1, w^{*} \in \partial\left\langle y^{*}, f\right\rangle(F(\bar{x})), x_{1}^{*} \in$ $\partial\left\langle w^{*}, F\right\rangle(\bar{x}), v^{*} \in \partial\left\langle z^{*}, g\right\rangle(G(\bar{x}))$, and $x_{2}^{*} \in \partial\left\langle v^{*}, G\right\rangle(\bar{x})$, one can find $v \in-N(\bar{x} ; \Omega)^{+}$ satisfying

$$
\begin{aligned}
\left\langle y^{*}, f \circ F\right\rangle(x)-\left\langle y^{*}, f \circ F\right\rangle(\bar{x}) & \geq\left\langle x_{1}^{*}, v\right\rangle, \\
\left\langle z^{*}, g \circ G\right\rangle(x)-\left\langle z^{*}, g \circ G\right\rangle(\bar{x}) & \geq\left\langle x_{2}^{*}, v\right\rangle \text { and } \\
\left\langle b^{*}, v\right\rangle & \leq\|x-\bar{x}\|, \forall b^{*} \in B_{X} .
\end{aligned}
$$

(ii) We say that $(f \circ F, g \circ G)$ is $K$-strictly $(K \times S)$-approximately generalized convex on $\Omega$ at $\bar{x} \in \Omega$ if for any $x \in \Omega \backslash\{\bar{x}\}, y^{*} \in K^{+}, z^{*} \in S^{+},\left\|y^{*}\right\|+\left\|z^{*}\right\|=1$, $w^{*} \in \partial\left\langle y^{*}, f\right\rangle(F(\bar{x})), x_{1}^{*} \in \partial\left\langle w^{*}, F\right\rangle(\bar{x}), v^{*} \in \partial\left\langle z^{*}, g\right\rangle(G(\bar{x}))$, and $x_{2}^{*} \in \partial\left\langle v^{*}, G\right\rangle(\bar{x})$, one can find $v \in-N(\bar{x} ; \Omega)^{+}$satisfying

$$
\begin{aligned}
\left\langle y^{*}, f \circ F\right\rangle(x)-\left\langle y^{*}, f \circ F\right\rangle(\bar{x}) & >\left\langle x_{1}^{*}, v\right\rangle, \\
\left\langle z^{*}, g \circ G\right\rangle(x)-\left\langle z^{*}, g \circ G\right\rangle(\bar{x}) & \geq\left\langle x_{2}^{*}, v\right\rangle \text { and } \\
\left\langle b^{*}, v\right\rangle & \leq\|x-\bar{x}\|, \forall b^{*} \in B_{X} .
\end{aligned}
$$

Remark 3.3. Given $\bar{x} \in \Omega$. In view of [8, Proposition 3.9], it can be observed that, if $\Omega$ is convex, $\left\langle y^{*}, f\right\rangle$ is convex on $\Omega$ for every $y^{*} \in K^{+},\left\langle z^{*}, g\right\rangle$ is convex on $\Omega$ for every $z^{*} \in S^{+},\left\langle w^{*}, F\right\rangle$ is convex on $\Omega$ for every $w^{*} \in \partial\left\langle y^{*}, f\right\rangle(F(\bar{x}))$ and $\left\langle v^{*}, G\right\rangle$ is convex on $\Omega$ for every $v^{*} \in \partial\left\langle z^{*}, g\right\rangle(G(\bar{x}))$, then $(f \circ F, g \circ G)$ is $(K \times S)$-approximately generalized convex on $\Omega$ at $\bar{x} \in \Omega$ with $v:=x-\bar{x}$ for each $x \in \Omega$. Besides, the $K$-strictly $(K \times S)$ approximately generalized convexity of $(f \circ F, g \circ G)$ on $\Omega$ at $\bar{x}$ will follows if, in addition, $\left\langle y^{*}, f\right\rangle$ is strictly convex on $\Omega$ for every $y^{*} \in K^{+} \backslash\{0\}$ and $F$ is injective on $\Omega$ (i.e., $\forall x_{1}, x_{2} \in$ $\left.\Omega, F\left(x_{1}\right)=F\left(x_{2}\right) \Rightarrow x_{1}=x_{2}\right)$. Furthermore, [8, Remark 3.10] indicated that the class of approximately generalized convex composite vector functions defined in Definition 3.3 is properly bigger than the one of convex vector functions.

we are now in a position to provide sufficient conditions for (weak) $e$-quasi efficient solutions of problem (P) under the satisfaction of the (strictly) approximately generalized convexity.

Theorem 3.2. Let $e \in K$ and assume that $\bar{x} \in C$ satisfies the e-approximate $K K T$ condition of problem $(\mathrm{P})$.

(i) If $(f \circ F, g \circ G)$ is $(K \times S)$-approximately generalized convex on $\Omega$ at $\bar{x}$, then $\bar{x} \in e-\mathcal{S}^{w}(\mathrm{P})$.

(ii) If $(f \circ F, g \circ G)$ is $K$-strictly $(K \times S)$-approximately generalized convex on $\Omega$ at $\bar{x}$, then $\bar{x} \in e-\mathcal{S}(\mathrm{P})$.

Proof. As $\bar{x} \in C$ is an $e$-approximate KKT point of problem (P), there exist $y^{*} \in K^{+} \backslash\{0\}, z^{*} \in$ $S^{+}, w^{*} \in \partial\left\langle y^{*}, f\right\rangle(F(\bar{x})), x_{1}^{*} \in \partial\left\langle w^{*}, F\right\rangle(\bar{x}), v^{*} \in \partial\left\langle z^{*}, g\right\rangle(\bar{x}), x_{2}^{*} \in \partial\left\langle v^{*}, G\right\rangle(\bar{x})$ and $b^{*} \in B_{X}$ such that $\left\|y^{*}\right\|+\left\|z^{*}\right\|=1$ and

$$
\begin{gathered}
-\left(x_{1}^{*}+x_{2}^{*}+\left\langle y^{*}, e\right\rangle b^{*}\right) \in N(\bar{x} ; \Omega), \\
\left\langle z^{*}, g(G(\bar{x}))\right\rangle=0 .
\end{gathered}
$$

To justify (i), we assume by contradiction that $\bar{x} \notin e-S^{w}(\mathrm{P})$, and consequently, there is $\hat{x} \in C$ such that $(f \circ F)(\hat{x})-(f \circ F)(\bar{x})+\|\hat{x}-\bar{x}\| e \in-i n t K$. It then follows from [22, Lemma 3.21] that

$$
\left.\left\langle y^{*},(f \circ F)(\hat{x})-(f \circ F)(\bar{x})\right\rangle+\|\hat{x}-\bar{x}\| e\right\rangle<0 .
$$


Due to the approximately generalized convexity of $(f \circ F, g \circ G)$ on $\Omega$ at $\bar{x}$, taking into account (3.15) together with the fact that $\left\langle y^{*}, e\right\rangle \geq 0$, we find $v \in-N(\bar{x} ; \Omega)^{+}$satisfying

$$
\begin{aligned}
0 & \leq\left\langle x_{1}^{*}, v\right\rangle+\left\langle x_{2}^{*}, v\right\rangle+\left\langle y^{*}, e\right\rangle\left\langle b^{*}, v\right\rangle \\
& \leq\left\langle y^{*}, f \circ F\right\rangle(\hat{x})-\left\langle y^{*}, f \circ F\right\rangle(\bar{x})+\left\langle z^{*}, g \circ G\right\rangle(\hat{x})-\left\langle z^{*}, g \circ G\right\rangle(\bar{x})+\left\langle y^{*}, e\right\rangle\|\hat{x}-\bar{x}\| .
\end{aligned}
$$

In addition, we have $\left\langle z^{*}, g(G(\hat{x}))\right\rangle \leq 0$ inasmuch as $g(G(\hat{x})) \in-S$. Taking (3.16) into account, we now invoke the second inequality in (3.18) to deduce that

$$
\left.0 \leq\left\langle y^{*},(f \circ F)(\hat{x})-(f \circ F)(\bar{x})\right\rangle+\|\hat{x}-\bar{x}\| e\right\rangle,
$$

which contradicts to (3.17), and so the proof of (i) has been established.

Next, we now prove (ii) by the method of contradiction and suppose that $\bar{x} \notin e-S(P)$. So, we can find $\tilde{x} \in C$ such that $(f \circ F)(\tilde{x})-(f \circ F)(\bar{x})+\|\tilde{x}-\bar{x}\| e \in-K \backslash\{0\}$, which in turn implies that $\tilde{x} \neq \bar{x}$ and

$$
\left\langle y^{*},(f \circ F)(\tilde{x})-(f \circ F)(\bar{x})+\|\tilde{x}-\bar{x}\| e\right\rangle \leq 0 .
$$

Proceeding similarly as in the proof of (i), we arrive at

$$
\left.0<\left\langle y^{*},(f \circ F)(\hat{x})-(f \circ F)(\bar{x})\right\rangle+\|\hat{x}-\bar{x}\| e\right\rangle,
$$

which is a contradiction to (3.19), and so the proof is complete.

\section{DUALITY FOR APPROXIMATE SOLUTIONS IN COMPOSITE VECTOR OPTIMIZATION}

In this section, we address a dual vector problem to the composite vector optimization problem (P) and examine converse and strong dualities assertions for approximate (weak) efficient solutions of $(\mathrm{P})$ and its dual which is formulated in the sense of Mond and Weir [33].

Given $e \in K$, we consider a dual vector program in connection with the problem $(\mathrm{P})$ as follows:

$$
\max _{K}\left\{\mathcal{L}\left(z, y^{*}, z^{*}\right):=(f \circ F)(z) \mid\left(z, y^{*}, z^{*}\right) \in C_{D}\right\},
$$

where the feasible set $C_{D}$ is given by

$$
\begin{aligned}
C_{D}:=\{ & \left(z, y^{*}, z^{*}\right) \in \Omega \times\left(K^{+} \backslash\{0\}\right) \times S^{+} \mid 0 \in \bigcup_{w^{*} \in \partial\left\langle y^{*}, f\right\rangle(F(z))} \partial\left\langle w^{*}, F\right\rangle(z) \\
& \left.+\bigcup_{v^{*} \in \partial\left\langle z^{*}, g\right\rangle(G(z))} \partial\left\langle v^{*}, G\right\rangle(z)+\left\langle y^{*}, e\right\rangle B_{X}+N(z ; \Omega),\left\langle z^{*}, g(G(z))\right\rangle \geq 0\right\} .
\end{aligned}
$$

The approximate efficient solutions for the dual vector problem (D) are defined in an analogous manner as for the primal problem $(\mathrm{P})$ stated in Definition 1.1.

\section{Definition 4.4.}

(i) We say that $\left(\bar{z}, \bar{y}^{*}, \bar{z}^{*}\right) \in C_{D}$ is an $e$-quasi efficient solution of problem (D), denoted by $\left(\bar{z}, \bar{y}^{*}, \bar{z}^{*}\right) \in e-\mathcal{S}(\mathrm{D})$, whenever

$$
\forall\left(z, y^{*}, z^{*}\right) \in C_{D}, \mathcal{L}\left(z, y^{*}, z^{*}\right)-\mathcal{L}\left(\bar{z}, \bar{y}^{*}, \bar{z}^{*}\right)-\left\|\left(z, y^{*}, z^{*}\right)-\left(\bar{z}, \bar{y}^{*}, \bar{z}^{*}\right)\right\| e \notin K \backslash\{0\} .
$$

(ii) One say that $\left(\bar{z}, \bar{y}^{*}, \bar{z}^{*}\right) \in C_{D}$ is a weak $e$-quasi efficient solution of problem (D), denoted by $\left(\bar{z}, \bar{y}^{*}, \bar{z}^{*}\right) \in e-\mathcal{S}^{w}(\mathrm{D})$, whenever

$$
\forall\left(z, y^{*}, z^{*}\right) \in C_{D}, \mathcal{L}\left(z, y^{*}, z^{*}\right)-\mathcal{L}\left(\bar{z}, \bar{y}^{*}, \bar{z}^{*}\right)-\left\|\left(z, y^{*}, z^{*}\right)-\left(\bar{z}, \bar{y}^{*}, \bar{z}^{*}\right)\right\| e \notin \operatorname{int} K \text {. }
$$

The next theorem describes strong duality relations for (weak) $e$-quasi efficient solutions of problem (P) and problem (D). 
Theorem 4.3 (Strong Duality). Let $e \in K$ and assume that (CQ) is satisfied at $\bar{x} \in e-\mathcal{S}^{w}(\mathrm{P})$. Then, there exists $\left(\bar{y}^{*}, \bar{z}^{*}\right) \in K^{+} \times S^{+}$such that $\left(\bar{x}, \bar{y}^{*}, \bar{z}^{*}\right) \in C_{D}$. Furthermore, the following statements hold:

(i) If $(f \circ F, g \circ G)$ is $(K \times S)$-approximately generalized convex on $\Omega$ at $z$ for all $z \in \Omega$, then $\left(\bar{x}, \bar{y}^{*}, \bar{z}^{*}\right) \in e-\mathcal{S}^{w}(\mathrm{D})$.

(ii) If $(f \circ F, g \circ G)$ is $K$-strictly $(K \times S)$-approximately generalized convex on $\Omega$ at $z$ for all $z \in \Omega$, then $\left(\bar{x}, \bar{y}^{*}, \bar{z}^{*}\right) \in e-\mathcal{S}(\mathrm{D})$.

Proof. In view of Theorem 3.1, it stems from $\bar{x} \in e-\mathcal{S}^{w}(\mathrm{P})$ that there exist $\bar{y}^{*} \in K^{+}$and $\bar{z}^{*} \in S^{+}$with $\left\|\bar{y}^{*}\right\|+\left\|\bar{z}^{*}\right\|=1$ such that

$\left\{\begin{array}{l}0 \in \bigcup_{w^{*} \in \partial\left\langle\bar{y}^{*}, f\right\rangle(F(\bar{x}))} \partial\left\langle w^{*}, F\right\rangle(\bar{x})+\bigcup_{v^{*} \in \partial\left\langle\bar{z}^{*}, g\right\rangle(G(\bar{x}))} \partial\left\langle v^{*}, G\right\rangle(\bar{x})+\left\langle y^{*}, e\right\rangle B_{X}+N(\bar{x} ; \Omega), \\ \left\langle\bar{z}^{*}, g(G(\bar{x}))\right\rangle=0 .\end{array}\right.$

Since the (CQ) is satisfied at $\bar{x}$, it follows that $\bar{y}^{*} \neq 0$. So, we conclude that $\left(\bar{x}, \bar{y}^{*}, \bar{z}^{*}\right) \in C_{D}$.

We first justify (i). Let $(f \circ F, g \circ G)$ be $(K \times S)$-approximately generalized convex on $\Omega$ at any $z \in \Omega$. Suppose on the contrary that $\left(\bar{x}, \bar{y}^{*}, \bar{z}^{*}\right) \notin e-\mathcal{S}^{w}(\mathrm{D})$. This means that there exists $\left(z, y^{*}, z^{*}\right) \in C_{D}$ such that $\mathcal{L}\left(z, y^{*}, z^{*}\right)-\mathcal{L}\left(\bar{x}, \bar{y}^{*}, \bar{z}^{*}\right)-\left\|\left(z, y^{*}, z^{*}\right)-\left(\bar{x}, \bar{y}^{*}, \bar{z}^{*}\right)\right\| e \in$ int $K$, which in turn is equivalent to the assertion

$$
(f \circ F)(z)-(f \circ F)(\bar{x})-\left\|\left(z, y^{*}, z^{*}\right)-\left(\bar{x}, \bar{y}^{*}, \bar{z}^{*}\right)\right\| e \in \operatorname{int} K,
$$

owing to the definition of $\mathcal{L}$. As $\left(z, y^{*}, z^{*}\right) \in C_{D}$, there exist $w^{*} \in \partial\left\langle y^{*}, f\right\rangle(F(z)), x_{1}^{*} \in$ $\partial\left\langle w^{*}, F\right\rangle(z), v^{*} \in \partial\left\langle z^{*}, g\right\rangle(z), x_{2}^{*} \in \partial\left\langle v^{*}, G\right\rangle(z)$ and $b^{*} \in B_{X}$ such that $\left\|y^{*}\right\|+\left\|z^{*}\right\|=1$, and

$$
\begin{gathered}
-\left(x_{1}^{*}+x_{2}^{*}+\left\langle y^{*}, e\right\rangle b^{*}\right) \in N(z ; \Omega), \\
\left\langle z^{*}, g(G(z))\right\rangle \geq 0 .
\end{gathered}
$$

On the one hand, it should be noted that $y^{*} \in K^{+} \backslash\{0\}$. So, taking [22, Lemma 3.21] into account, we derive from (4.20) that

$$
\left\langle y^{*},(f \circ F)(\bar{x})+\left\|\left(z, y^{*}, z^{*}\right)-\left(\bar{x}, \bar{y}^{*}, \bar{z}^{*}\right)\right\| e-(f \circ F)(z)\right\rangle<0 .
$$

By virtue of the approximately generalized convexity of $(f \circ F, g \circ G)$ on $\Omega$ at $z \in \Omega$, we deduce from (4.21) that, for such $\bar{x}$, there exists $v \in-N(z ; \Omega)^{+}$such that

$$
\begin{aligned}
0 & \leq\left\langle x_{1}^{*}, v\right\rangle+\left\langle x_{2}^{*}, v\right\rangle+\left\langle y^{*}, e\right\rangle\left\langle b^{*}, v\right\rangle \\
\text { (4.24) } & \leq\left\langle y^{*}, f \circ F\right\rangle(\bar{x})-\left\langle y^{*}, f \circ F\right\rangle(z)+\left\langle z^{*}, g \circ G\right\rangle(\bar{x})-\left\langle z^{*}, g \circ G\right\rangle(z)+\left\langle y^{*}, e\right\rangle\|\bar{x}-z\|,
\end{aligned}
$$

where we should remind that $\left\langle y^{*}, e\right\rangle \geq 0$. Due to the feasibility of $\bar{x}$, we have $\left\langle z^{*}, g \circ\right.$ $G\rangle(\bar{x}) \leq 0$. This together with (4.22) and (4.24) in turn gives us that

$$
0 \leq\left\langle y^{*},(f \circ F)(\bar{x})+\|\bar{x}-z\| e-(f \circ F)(z)\right\rangle .
$$

Now, combining (4.23) and (4.25), we arrive at

$$
\left\langle y^{*}, e\right\rangle\left\|\left(z, y^{*}, z^{*}\right)-\left(\bar{x}, \bar{y}^{*}, \bar{z}^{*}\right)\right\|<\left\langle y^{*}, e\right\rangle\|\bar{x}-z\|,
$$

which is a contradiction. Hence, $\left(\bar{x}, \bar{y}^{*}, \bar{z}^{*}\right) \in e-\mathcal{S}^{w}(\mathrm{D})$.

Now, we prove (ii). Let $(f \circ F, g \circ G)$ be $K$-strictly $(K \times S)$-approximately generalized convex on $\Omega$ at any $z \in \Omega$ and assume that $\left(\bar{x}, \bar{y}^{*}, \bar{z}^{*}\right) \notin e-\mathcal{S}(\mathrm{D})$. Then, one can find $\left(z, y^{*}, z^{*}\right) \in C_{D}$ such that

$$
(f \circ F)(z)-(f \circ F)(\bar{x})-\left\|\left(z, y^{*}, z^{*}\right)-\left(\bar{x}, \bar{y}^{*}, \bar{z}^{*}\right)\right\| e \in K \backslash\{0\} .
$$

Observe that (4.26) infers to $\bar{x} \neq z$. Moreover, we also have (4.21) and (4.22) by the dual feasibility of $\left(z, y^{*}, z^{*}\right)$. Now, it holds by (4.26) that

$$
\left\langle y^{*},(f \circ F)(\bar{x})+\left\|\left(z, y^{*}, z^{*}\right)-\left(\bar{x}, \bar{y}^{*}, \bar{z}^{*}\right)\right\| e-(f \circ F)(z)\right\rangle \leq 0,
$$


and, by definition of the dual cone and the $K$-strictly $(K \times S)$-approximately generalized convexity of $(f \circ F, g \circ G)$ on $\Omega$ at $z \in \Omega$, we conclude by (4.21) that for $\bar{x}$ above, one can find $v \in-N(z ; \Omega)^{+}$such that

$$
\begin{aligned}
0 & \leq\left\langle x_{1}^{*}, v\right\rangle+\left\langle x_{2}^{*}, v\right\rangle+\left\langle y^{*}, e\right\rangle\left\langle b^{*}, v\right\rangle \\
& <\left\langle y^{*}, f \circ F\right\rangle(\bar{x})-\left\langle y^{*}, f \circ F\right\rangle(z)+\left\langle z^{*}, g \circ G\right\rangle(\bar{x})-\left\langle z^{*}, g \circ G\right\rangle(z)+\left\langle y^{*}, e\right\rangle\|\bar{x}-z\| .
\end{aligned}
$$

Since as $\left\langle z^{*}, g \circ G\right\rangle(x) \leq 0$ shown above, (4.22) together with (4.28) gives us that

$$
0<\left\langle y^{*},(f \circ F)(\bar{x})+\|\bar{x}-z\| e-(f \circ F)(z)\right\rangle .
$$

This together with (4.27) establishes a contradiction. So, $\left(\bar{x}, \bar{y}^{*}, \bar{z}^{*}\right) \in e-\mathcal{S}(\mathrm{D})$.

Remark 4.4. It is worth noting that the (CQ) imposed in Theorem 4.3 plays a key role to confirm the existence of multiplier vectors $\left(\bar{y}^{*}, \bar{z}^{*}\right) \in K^{+} \times S^{+}$corresponding to $\bar{x} \in$ $e-\mathcal{S}^{w}(\mathrm{P})$ so that $\left(\bar{x}, \bar{y}^{*}, \bar{z}^{*}\right) \in C_{D}$. Note also that the conclusion of Theorem 4.3 may go awry if the approximate generalized convexity of $(f \circ F, g \circ G)$ on $\Omega$ at $\bar{x}$ is violated. These facts will demonstrate in Example 4.2 and Example 4.3, respectively.

Example 4.2. Let $\Omega:=\mathbb{R}, K:=\mathbb{R}_{+}^{2}$ and $S:=[0,+\infty)$. Considering the problem (P), where $F(x):=(x+1, x+1), x \in \mathbb{R}, f(w):=\left(w_{1}, w_{2}+1\right), w:=\left(w_{1}, w_{2}\right) \in \mathbb{R}^{2}, G(x):=x+1, x \in \mathbb{R}$, $g(v):=v^{2}, v \in \mathbb{R}$. It is easy to verify that $C=\{-1\}$, and hence, $\bar{x}:=-1 \in e-\mathcal{S}^{w}(\mathrm{P})(=$ $e-\mathcal{S}(\mathrm{P}))$ with any given $e \in K$; see e.g., [9, Example 4.3]. It could be convenient to observe that $(f \circ F, g \circ G)$ is $(K \times S)$-approximately generalized convex on $\Omega$ at $y$ for all $y \in \Omega$. We now examine the dual vector problem (D) with $e:=\left(\frac{1}{2}, \frac{1}{2}\right) \in K$. We can verify that there does not exist $\left(y^{*}, z^{*}\right) \in K^{+} \times S^{+}$fulfilling $\left(\bar{x}, y^{*}, z^{*}\right) \in C_{D}$. This means that the conclusion of Theorem 4.3 fails to hold in this case. The reason is that the (CQ) at $\bar{x}$ was violated.

Example 4.3. Let $\Omega:=\mathbb{R}_{+}, K:=\mathbb{R}_{+}^{2}$ and $S:=[0,+\infty)$. We consider the problem (P), where $F(x):=(x-2)^{3}+1, x \in \mathbb{R}, f(w):=\left(w, w^{2}+2 w-1\right), w \in \mathbb{R}, G(x):=x-1, x \in \mathbb{R}$, $g(v):=-|v|, v \in \mathbb{R}$. Then, $C:=[1,+\infty)$, and let us select $\bar{x}:=1 \in C$. It can be verified that $\bar{x} \in e-\mathcal{S}^{w}(\mathrm{P})$ for any $e \in K$; see e.g., [9, Example 4.4] and the (CQ) is satisfied at $\bar{x}$.

Now, let us consider the dual problem (D). Theorem 4.3 yields that there exist $\bar{y}^{*}:=$ $\left(\bar{y}_{1}^{*}, \bar{y}_{2}^{*}\right) \in K^{+}$and $\bar{z}^{*} \in S^{+}$such that $\left(\bar{x}, \bar{y}^{*}, \bar{z}^{*}\right) \in C_{D}$. Choosing $z:=2 \in \Omega, y^{*}:=$ $(1,1) \in K^{+}$, and $z^{*}:=0 \in S^{+}$, we can see that $\left(z, y^{*}, z^{*}\right) \in C_{D}$. By selecting $e:=$ $\left(e_{1}, e_{2}\right) \in K$ with $e_{1}<\frac{1}{\left\|\left(z, y^{*}, z^{*}\right)-\left(\bar{x}, \bar{y}^{*}, \bar{z}^{*}\right)\right\|}, e_{2}<\frac{3}{\left\|\left(z, y^{*}, z^{*}\right)-\left(\bar{x}, \bar{y}^{*}, \bar{z}^{*}\right)\right\|}$, it can be checked that $(f \circ F)(z)-(f \circ F)(\bar{x})-\left\|\left(z, y^{*}, z^{*}\right)-\left(\bar{x}, \bar{y}^{*}, \bar{z}^{*}\right)\right\| e \in$ int $K$, which is nothing else than $\left(\bar{x}, \bar{y}^{*}, \bar{z}^{*}\right) \notin e-\mathcal{S}^{w}(\mathrm{D})$. This shows that the conclusion of Theorem 4.3 fails since $(f \circ F, g \circ G)$ is not $(K \times S)$-approximately generalized convex on $\Omega$ at $z$.

We close this paper by presenting converse-like duality relations for (weak) $e$-quasi efficient solutions of problem $(\mathrm{P})$ and problem $(\mathrm{D})$.

Theorem 4.4. Let $\left(\bar{x}, y^{*}, z^{*}\right) \in C_{D}$ be such that $\bar{x} \in C$.

(i) If $(f \circ F, g \circ G)$ is $(K \times S)$-approximately generalized convex on $\Omega$ at $\bar{x}$, then $\bar{x} \in e-\mathcal{S}^{w}(\mathrm{P})$.

(ii) If $(f \circ F, g \circ G)$ is $K$-strictly $(K \times S)$-approximately generalized convex on $\Omega$ at $\bar{x}$, then $\bar{x} \in e-\mathcal{S}(\mathrm{P})$.

Proof. Since $\left(\bar{x}, y^{*}, z^{*}\right) \in C_{D}$, we have that $y^{*} \in K \backslash\{0\}, z^{*} \in S^{+}$and

$$
\left\{\begin{array}{l}
0 \in \bigcup_{w^{*} \in \partial\left\langle y^{*}, f\right\rangle(F(\bar{x}))} \partial\left\langle w^{*}, F\right\rangle(\bar{x})+\bigcup_{v^{*} \in \partial\left\langle z^{*}, g\right\rangle(G(\bar{x}))} \partial\left\langle v^{*}, G\right\rangle(\bar{x})+\left\langle y^{*}, e\right\rangle B_{X}+N(\bar{x} ; \Omega), \\
\left\langle z^{*}, g(G(\bar{x}))\right\rangle \geq 0 .
\end{array}\right.
$$


Let us notice, as $\bar{x} \in C$, i.e., $(g \circ G)(\bar{x}) \in-S$, that $\left\langle z^{*}, g(G(\bar{x}))\right\rangle \leq 0$. Consequently, $\left\langle z^{*}, g(G(\bar{x}))\right\rangle=0$. Note in addition that as $y^{*} \in K \backslash\{0\}$, we can put $\bar{y}^{*}:=\frac{y^{*}}{\left\|y^{*}\right\|+\left\|z^{*}\right\|}, \bar{z}^{*}:=$ $\frac{z^{*}}{\left\|y^{*}\right\|+\left\|z^{*}\right\|}$, and so, $\bar{y}^{*} \in K^{+} \backslash\{0\}, \bar{z}^{*} \in S^{+}$and $\left\|\bar{y}^{*}\right\|+\left\|\bar{z}^{*}\right\|=1$. It can be observed that (4.29) also holds if $y^{*}$ and $z^{*}$ are replaced by $\bar{y}^{*}$ and $\bar{z}^{*}$, respectively. So, in view of Definition 3.2, we arrive at the conclusion that $\bar{x}$ satisfies the $e$-approximate KKT condition of problem $(\mathrm{P})$. The rest of the proof follows by applying Theorem 3.1.

Acknowledgement: This research was partially supported by the Thailand Research Fund through the Royal Golden Jubilee Ph. D. Program (Grant No. PHD/0220/2556) and Naresuan University.

\section{REFERENCES}

[1] Boţ, R. I.; Hodrea, I. B.; Wanka, G. Optimality conditions for weak efficiency to vector optimization problems with composed convex functions. Cent. Eur. J. Math. 6 (2008), no. 3, 453-468.

[2] Boţ, R. I.; Vargyas, E.; Wanka, G. Conjugate duality for multiobjective composed optimization problems. Acta. Math. Hung. 116 (2007), no. 3, 177-196.

[3] Chinchuluun, A.; Pardalos, P. M. A survey of recent developments in multiobjective optimization. Ann Oper Res. 154 (2007), 29-50.

[4] Chuong, T. D.; Kim, D. S. Approximate solutions of multiobjective optimization problems. Positivity 20 (2016), no. 1, 187-207.

[5] Chuong, T. D. Optimality conditions for nonsmooth multiobjective bilevel optimization problems. Ann. Oper. Res. 251 (2017), 73-87.

[6] Chuong, T. D. Linear matrix inequality conditions and duality for a class of robust multiobjective convex polynomial programs. SIAM J. Optim. 28 (2018), no. 3, 2466-2488.

[7] Chuong, T. D. Optimality and duality in nonsmooth conic vector optimization. J. Optim. Theory Appl. 183 (2019), 471-489.

[8] Chuong, T. D. Optimality and duality in nonsmooth composite vector optimization and applications. Ann. Oper. Res. (2019), https://doi.org/10.1007/s10479-019-03349-1.

[9] Choung, T. D. Approximate solutions in nonsmooth and nonconvex cone constrained vector optimization Ann. Oper. Res. (2020), https://doi.org/10.1007/s10479-020-03740-3.

[10] Durea, M.; Dutta, J.; Tammer, Chr. Lagrange multipliers for $\varepsilon$-Pareto solutions in vector optimization with nonsolid cones in Banach spaces. J. Optim. Theory Appl. 145 (2010), no. 1, 196-211.

[11] Ekeland, I. On the variational principle. J. Math. Anal. Appl. 47 (1974), 324-353.

[12] Engau, A.; Wiecek, M. M. Cone characterizations of approximate solutions in real vector optimization. J. Optim. Theory Appl. 134 (2007), no. 3, 499-513.

[13] Gao, Y.; Hou, S. H.; Yang, X. M. Existence and optimality conditions for approximate solutions to vector optimization problems. J. Optim. Theory Appl. 152 (2012), 97-120.

[14] Ghaznavi, M.; Akbarib, F.; Khorram, E. Optimality conditions via a unified direction approach for (approximate) efficiency in multiobjective optimization. Optim. Methods Softw. (2019), https: / / doi.org/10.1080/10556788.2019.1571589.

[15] Ghaznavi-Ghosoni, B. A.; Khorram, E.; Soleimani-Damaneh, M. Scalarization for characterization of approximate strong/weak/proper efficiency in multi-objective optimization. Optimization 62 (2013), no. 6, 703-720.

[16] Govil, M. G. and Mehra, A., E-optimality formultiobjective programming on a Banach space, Eur. J. Opera. Res., 157 (2004), no. 1, 106-112.

[17] Gutiérrez, C.; Huerga, L.; Jiménez, B. ; Novo, V. Approximate solutions of vector optimization problems via improvement sets in real linear spaces. J. Glob. Optim. 70 (2018), no. 4, 875-901.

[18] Gutiérrez, C.; Jiménez, B.; Novo, V. A unified approach and optimality conditions for approximate solutions of vector optimization problems. SIAM J. Optim. 17 (2006), no. 3, 688-710.

[19] Gutiérrez, C.; Jiménez, B.; Novo, V. Optimality conditions via scalarization for a new $\epsilon$-efficiency concept in vector optimization problems. Eur. J. Opera. Res. 201 (2010), no. 1, 11-22.

[20] Gupta, D.; Mehra, A. A new notion of quasi efficiency in vector optimization. Pac. J. Optim. 8 (2012), no. 2, 217-230.

[21] Huang, X. X. Optimality conditions and approximate optimality conditions in locally Lipschitz vector optimization Optimization 51 (2002), no. 2, 309-321.

[22] Jahn, J., Vector Optimization. Theory, Applications and Extensions, Springer, Berlin, 2004. 
[23] Jeyakumar, V.; Yang, X. Q. Convex composite multi-objective nonsmooth programming. Math. Program. 59 (1993), no. 3, 325-343.

[24] La Torre, D. Necessary optimality conditions for nonsmooth vector optimization problems. Math. Model. Analysis 8 (2003), no. 2, 165-174.

[25] Lee, J. H.; Lee, G. M. On optimality conditions and duality theorems for robust semi-infinite multiobjective optimization problems. Ann. Oper. Res. 269 (2018), no. 1-2, 419-438.

[26] Liu, J. C. $\varepsilon$-duality theorem of nondifferentiable nonconvex multiobjective programming. J. Optim. Theory Appl. 69 (1991), no. 1, 153-167.

[27] Liu, J. C. e-Pareto optimality for nondifferentiable multiobjective programing via penalty function. J. Math. Anal. Appl. 198 (1996), 248-261.

[28] Loridan, P. $\varepsilon$-solutions in vector minimization problems. J. Optim. Theory Appl. 43 (1984), no. 2, $265-276$.

[29] Luc, D. T. Theory of Vector Optimization. Lecture Notes in Economics and Mathematical Systems, Springer, Berlin, 1989.

[30] Minami, M. Weak Pareto-optimal necessary conditions in a nondifferentiable multiobjective program on a Banach space. J. Optim. Theory Appl. 41 (1983), no. 3, 451-461.

[31] Mishra, S. K. Lagrange multipliers saddle points and scalarizations in composite multiobjective nonsmooth programming. Optimization 38 (1996), no. 2, 93-105.

[32] Mishra, S. K.; Mukherjee, R. N. Generalized convex composite multi-objective nonsmooth programming and conditional proper efficiency. Optimization 34 (1995), no. 1, 53-66.

[33] Mond, B.; Weir, T. Generalized concavity and duality. In S. Schaible \& W. T. Ziemba (Eds.), Generalized concavity in optimization and economics Academic Press., (1981), 263-279.

[34] Mordukhovich B. S. Variational Analysis and Generalized Differentiation. I. Basic Theory, Springer, Berlin, 2006.

[35] Mordukhovich, B. S. Variational Analysis and Applications, Springer Monographs in Mathematics, Springer, Berlin, 2018.

[36] Reddy, L. V.; Mukherjee, R. N. Composite nonsmooth multiobjective programs with $V$ - $\rho$-invexity. J. Math. Anal. Appl. 235 (1999), no. 2, 567-577.

[37] Rockafellar, R. T. Convex analysis. Princeton, Princeton University Press, New Jersey, 1970.

[38] Sach, P. H.; Kim, D. S.; Lee, G. M. Generalized convexity and nonsmooth problems of vector optimization. J. Glob. Optim. 31 (2005), no. 3, 383-403.

[39] Sawaragi, Y.; Nakayama, H.; Tanino, T. Theory of multiobjective optimization. Mathematics in science and engineering, Academic Press, Inc., Orlando, 1985.

[40] Son, T. Q.; Kim, D. S. $\varepsilon$-mixed type duality for nonconvex multiobjective programs with an infinite number of constraints. J. Glob. Optim. 57 (2013), no. 2, 447-465.

[41] Suneja, S. K.; Sharma, S.; Yadav, P. Generalized higher-order cone-convex functions and higher-order duality in vector optimization. textit Ann. Oper. Res. 269 (2018), no. 1-2, 709-725.

[42] Tang, L. P.; Zhao, K. Q. Optimality conditions for a class of composite multiobjective nonsmooth optimization problems. J. Glob. Optim. 57 (2013), no. 2, 399-414.

\author{
${ }^{1}$ Department OF MATHEMATics \\ NARESUAN UNIVERSITY \\ FACUlTy OF SCIENCE, PhitsAnUlOK, 65000, ThAilAnd \\ E-mail address: ballthanat@hotmail.com ( $T$. Sirichunwijit) \\ E-mail address: rabianw@nu.ac.th (R. Wangkeeree) \\ E-mail address: nithirats@hotmail.com (N. Sisarat) \\ 2 Research Center for Academic Excellence in Mathematics, \\ NARESUAN UNIVERSITY, THAILAND
}

